\title{
Unexpected hematoma due to heparin prophylaxis in transverse rectus abdominis musculocutaneous flap reconstruction
}

\author{
Ayaz Shaikh MD, James R Bain MD MSc FRCSC \\ Department of Surgery, Division of Plastic Surgery, McMaster University, Hamilton, Ontario
}

\begin{abstract}
A Shaikh, JR Bain. Unexpected hematoma due to heparin prophylaxis in transverse rectus abdominis musculocutaneous flap reconstruction. Can J Plast Surg 2001;9(1):29-31.

Prophylaxis with standard low dose subcutaneous heparin has been shown to significantly decrease the risk of deep vein thrombosis and pulmonary embolism, without major bleeding complications. The present report describes a case of unexpected hematoma formation, possibly secondary to subcutaneous low dose heparin injection that was administered in the anterior abdominal wall in a patient who had previously undergone a transverse rectus abdominis musculocutaneous flap reconstruction.
\end{abstract}

Key Words: Complications; Deep vein thrombosis prophylaxis; Hematoma; Low dose heparin; Transverse rectus abdominis musculocutaneous flap

\section{Hématome inattendu consécutif à l'héparinoprophylaxie dans la reconstruction d'un lambeau musculo-cutané du droit transverse de l'abdomen}

RÉSUMÉ : La prophylaxie au moyen de faibles doses standard d'héparine sous-cutanée s'est révélée apte à réduire significativement le risque de thrombose veineuse profonde et d'embolie pulmonaire, sans complications hémorragiques majeures. On décrit ici un cas d'hématome inattendu, possiblement consécutif à l'administration d'une injection sous-cutanée d'héparine à faible dose administrée dans la paroi abdominale antérieure chez un patient qui venait de subir une reconstruction de lambeau musculo-cutané du droit transverse abdominal.

$\mathrm{P}$ rophylactic heparin came into widespread use after low dose or mini-dose heparin (low dose unfractionated heparin [LDUH]) was found to be effective in significantly reducing the risk of deep vein thrombosis (DVT) and pulmonary embolism, without major bleeding complications. This regimen is the standard for comparison with other methods, and it is the mainstay in moderate and high risk general surgery patients (1). It is thought to work because heparin concentrations that are too small to block the extension of preformed thrombi can prevent the activation of clotting factors that are high in the intrinsic clotting cascade from leading to thrombin production and thrombus formation.

Usually, $5000 \mathrm{U}$ heparin is given subcutaneously $2 \mathrm{~h}$ before surgery, and 8 to $12 \mathrm{~h}$ after surgery for five to seven days. Although it is thought to be very safe, low dose heparin prophylaxis increased clinically minor surgical bleeding from
$3.8 \%$ to $5.9 \%$ in patients or control groups, as shown by a meta-analysis of multiple trials (2).

Other pharmacological venous thrombosis prevention regimens consist of adjusted dose subcutaneous heparin, ultra low dose heparin, heparin or dihydroergotamine, low molecular weight heparin and heparinoids, mini-dose heparin, low or moderate dose perioperative warfarin and dextran 40/70 Da. In addition, venous flow accelerations, preoperative electric calf stimulation, graded pressure stockings and external intermittent calf or thigh compression have been used to decrease stasis.

The regimen used in the present study for DVT prophylaxis in patients undergoing breast reconstruction consisted of a preoperative subcutaneous injection of $5000 \mathrm{U}$ heparin, followed by $5000 \mathrm{U}$ heparin injected twice daily until the patient was ambulating well. Traditionally, common sites 
of injection are the anterior abdominal wall and the lateral thigh.

Heparin levels can be maintained at relatively stable levels for $24 \mathrm{~h}$ with twice daily subcutaneous injections. Heparin levels peak in 3 to $4 \mathrm{~h}$. This rationale is the basis for the protocol used at the Division of Plastic Surgery, McMaster University, Hamilton, Ontario.

A case of a hemorrhagic complication secondary to the use of prophylactic low dose heparin is described.

\section{CASE PRESENTATION}

A 69-year-old woman was scheduled to undergo a unilateral pedicled transverse rectus abdominis musculocutaneous (TRAM) flap reconstruction of the left breast. A modified radical mastectomy had been performed on the same breast eight years earlier.

The patient's past medical history consisted of coronary heart disease and gastroesophageal reflux disease. The surgical history included a hysterectomy through a Pfannenstiel's incision and an appendectomy in the distant past. Her medications consisted of atenolol, nitroglycerine spray, acetylsalicylic acid (Aspirin; Bayer, Canada) and omeprazole (Prilosec; AstraZeneca, Canada). The patient discontinued the use of Aspirin one week before surgery.

Preoperative examination revealed a mild rectus diastasis that was not considered to be significant. No other abnormal physical findings were observed.

According to routine preoperative orders, $5000 \mathrm{U}$ heparin was given subcutaneously. The patient received the dose in the anterior abdominal wall approximately $1 \mathrm{~h}$ before the surgery.

After an uneventful anesthesia induction, the procedure was performed as follows. The left mastectomy scar was excised and the defect was recreated by elevating skin flaps superficial to the pectoralis major, down to the inframammary fold. On raising the flap, an approximately $2 \mathrm{~cm}^{2}$ area of bruising and a small hematoma were noticed at the subcutaneous heparin injection site. The right unilateral pedicle TRAM flap was raised. Zone 4 was discarded. Zone 3 was de-epithelialized, and the flap was inset. No abnormal bleeding was noticed in the bed, mastectomy defect or flap donor site. Drains were placed in the usual fashion (one in the breast and two in the abdomen).

As part of the routine postoperative orders, hourly flap checks, which involved monitoring for capillary refill, colour, temperature and appearance were done. Postoperative medications did not include any anticoagulations or nonsteroidal anti-inflammatory drugs; however, the patient was continued on our low dose heparin protocol, $5000 \mathrm{U}$ injected subcutaneously twice daily.

Approximately $6 \mathrm{~h}$ after surgery, there was some transient difference in temperature with the control (contralateral) breast; however, the flap exhibited bleeding to needle stick puncture and remained warm, although somewhat pale in appearance.

One day after surgery, the capillary refill rate was found to be sluggish, but no apparent hematoma or swelling was evi- dent. There was bright red and brisk bleeding to needle stick centrally and over the medial aspect; however, there was no bleeding from the lateral part of the flap. The temperature gradient was within one degree of control (flap 33 to $35^{\circ} \mathrm{C}$, control 34 to $36^{\circ} \mathrm{C}$ ), and localized zone 3 fat necrosis was one of the considerations.

Operative site dressings were removed, and all systemic parameters were optimized, including measures such as a warming blanket, hydration and transfusion for a hemoglobin concentration of $8.1 \mathrm{~g} / \mathrm{L}$.

In the following $6 \mathrm{~h}$, the anterior chest wall became progessively firm and swollen, and temperatures dropped precipitously. The patient was taken urgently to the operating room. The entire incision at the superior aspect of the inset had to be released progressively. A large hematoma was evacuated from the axillary tail region, it also extended superiorly with the undermined chest wall pocket just superficial to the pectoralis major. An area of fat necrosis was noticed along the superior-medial aspect of the flap and was trimmed. Overall, the flap was trimmed back to brisk, bleeding, viable tissue by removing approximately $290 \mathrm{~g}$ of tissue. The underlying rectus abdominis muscle required only minimal excision of the devitalized muscle from the very distal aspect. The deep inferior epigastric artery and its venae commitantes were identified, and found to be securely ligated. No specific bleeding points were noticed. There was minimal skin loss, and the flap was inset in the standard fashion, although there was some loss of fullness, primarily along the superior aspect.

The patient had to undergo further revision six weeks later when the superior part of the lateral segment was found to be necrotic. The skin was elevated off the flap, and it was debrided back to viable tissue. The upper skin flap was advanced to cover the remaining healthy TRAM flap tissue, both superiorly and laterally. In the entire process, about $25 \%$ of the TRAM flap was excised. Overall, there was some volume discrepancy, but the shape and contour of the breast were acceptable. A balancing reduction mammoplasty with a nipple areola reconstruction is planned for this patient.

\section{DISCUSSION}

The unexpected hematoma formation was hypothesized to be secondary to the subcutaneous heparin that the patient received in the anterior abdominal wall before surgery. The $5000 \mathrm{U}$ that were meant for distribution in a $70 \mathrm{~kg}$ patient were distributed in a $3 \mathrm{~kg}$ flap.

Heparin is cleared by a biphasic pattern (ie, saturable and nonsaturable mechanisms). The initial rapid phase of clearance is consistent with a combination of equilibrium of heparin in plasma, and binding and uptake by endothelial and phagocytic cells, while the second phase reflects renal clearance. Therefore, clearance is dose dependent because larger doses would saturate the binding sites much faster (2). It was also hypothesized that excessive bleeding was not encountered during the procedure because the heparin distribution could be slow secondary to the flap being cold, hypoperfused and vasoconstricted during elevation as a part of the classic 
inflammatory response during surgical manoeuvring. In addition, after subcutaneous injection of heparin, peak levels are not obtained immediately but after 3 to $4 \mathrm{~h}$. Thus, the site of subcutaneous administration of heparin may have a bearing on the risk of major surgical bleeding and hematoma formation. No reports of studies linking the site of subcutaneous injection of prophylactic LDUH to the risk of hematoma formation in TRAM flap patients were found in the literature. However, a prospective, controlled, randomized clinical trial of patients undergoing elective inguinal hernia repair showed that the arm was preferred to the abdominal wall as the injection site for LDUH with regard to hematoma formation, which is significantly lower in patients who received LDUH in the arm (2.5\%) compared with those who received the injection in the abdominal wall (22.5\%) (3).

\section{CONCLUSIONS}

Contrary to the prevalent traditional belief that DVT prophylaxis with standard LDUH subcutaneously does not cause clinically significant bleeding, it was a cause of significant postoperative bleeding, unexpected hematoma formation and morbidity of the patient in the present case report.

Research by the authors for optimal DVT prophylaxis re- vealed studies showing low molecular weight heparin to be equally efficacious but superior in safety to LDUH, as far as wound hematoma formation is concerned $(4,5)$.

Due to an unusual complication described in the present paper and the literature that supports variable hematoma formation depending upon site of injection, McMaster University has made changes to its protocol to clearly delineate the site of injection of heparin (ie, the lateral arm).

\section{REFERENCES}

1. 5th ACCP Consensus Conference on Antithrombotic Therapy (1998): Summary Recommendations. Chest 1998;114(Suppl 5):439S-769S.

2. Colman RW, Hirsch J, Marder VJ, EW Salzman, eds. Hemostasis and Thrombosis: Basic Principles and Clinical Practice, 3rd edn. Philadelphia: Lippincott Williams \& Wilkins, 1994.

3. De Lange $\mathrm{S}$. Choice of injection site for low dose heparin in inguinal herniorrhaphy. Br J Surg 1982;69:23-45.

4. Kakkar VV, Beckl O, Boneu B, et al. Efficacy and safety of a low-molecular-weight-heparin and standard unfractionated heparin for prophylaxis of postoperative venous thromboembolism: European multicentre trial. World J Surg 1997;21:2-8; discussion 8-9.

5. Koch A, Bouges S, Zeigler S, et al. Low molecular weight heparin and unfractionated heparin in thrombosis prophylaxis after major surgical intervention: Update of previous meta-analysis. Br J Surg 1997;85:750-9. 